\title{
MICRO BALLOON ACTUATORS FOR AERODYNAMIC CONTROL
}

\author{
C. Grosjean, G.B. Lee*, W. Hong*, Y.C. Tai, C.M. Ho* \\ Caltech Micromachining Laboratory \\ Electrical Engineering 136-93 \\ Pasadena, CA 91125, USA \\ *Mechanical and Aerospace Engineering \\ University of California, Los Angeles \\ Los Angeles, CA 90095, USA
}

\begin{abstract}
A robust, large-force, large-deflection micro balloon actuator for aerodynamic (maneuvering) control of transonic aircraft has been developed. Using a novel process, high yield linear arrays of silicone balloons on a robust silicon substrate have been fabricated that can deflect vertically in excess of one $\mathrm{mm}$. Balloon actuators have been tested under cyclic conditions to assess reliability. The actuators have been characterized in a wind tunnel to assess their suitability as aerodynamic control surfaces and flight-tested on a jet fighter to assess their resistance to varied temperatures and pressures at high velocity.
\end{abstract}

\section{INTRODUCTION}

Previously, magnetically actuated surface and bulk micromachined flaps have been used for delta wing control surfaces [1]. This is fluid dynamically feasible because the similarity in length scales of the actuators and the boundary layer before separation at the leading edge of a wing allows the use of small surface perturbations to generate large aerodynamic control moments (pitch, yaw, and roll) through fluid amplification. However, these actuators were designed only for wind tunnel testing $(<50 \mathrm{~m} / \mathrm{s})$ and are not robust enough for flight tests on real aircraft or high speed $(>50 \mathrm{~m} / \mathrm{s})$ wind tunnel testing as the beams break. More robust beams would require very large magnetic forces and the resulting structures would still suffer from impacts during flight. For practical aircraft, requirements for a microactuator control surface then have to include robustness (e.g. resistance to particles), large deflection and load, low power consumption, surface conformability, and small size along the thickness of the wing. Pneumatically driven silicone membranes $[2,3,4]$ satisfy the first four requirements, but it can be difficult to design robust membranes that have small dead areas, as the frame can be very large.

We have fabricated large deflection silicone membranes [4] using anisotropic etching to form a free-standing membrane, but a large area was wasted due to the angular nature of the etching. Even if $\langle 110\rangle$ wafers and $\mathrm{KOH}$ or DRIE were used to form vertical sidewall cavities, the resulting structure would be very fragile if the membrane area was maximized. Some work has been done with photoresist sacrificial layers [3], but we have found that acetone attacks our silicone and photoresist developer is very slow to undercut large ( $\mathrm{mm}$ scale) structures. We have since developed a new process using Parylene $\mathrm{C}$ as a release layer for fabricating large silicone rubber membranes without the use of large bulk micromachined cavities, hence resulting in a robust frame. This novel technique takes advantage of the poor adhesion between silicone rubber and Parylene.

\section{DESIGN}

The desired geometry of the microactuators for wind tunnel testing and flight testing on a scale airfoil model were strips two to three $\mathrm{mm}$ wide, and stackable end to end for a total length as necessary depending on the wing. A vertical deflection on the order of one to two $\mathrm{mm}$ was needed for sufficient interaction with the boundary layer. Strips of actuators would then be

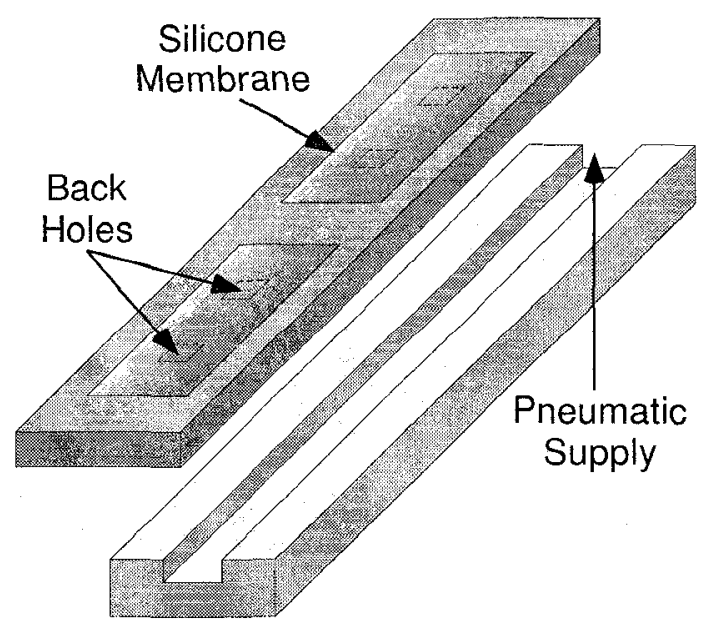

Fig. 1 Assembly of Actuator to Backing Strip 
attached to a suitable backing strip and pneumatically actuated. It was decided to put the groove for air flow in the backing strip so as to make the silicon wafer less prone to cracking during fabrication and the final actuator strips as robust as possible.

Earlier work with silicone membranes for valves [4] demonstrated that large deflection $(1.5 \mathrm{~mm})$ was achievable with small square silicone membranes, but they underwent plastic deformation and failed rapidly at high pressures. A first generation of micro balloon actuators was fabricated with dimensions as shown in Fig. 2. These actuators could actuate about one $\mathrm{mm}$ vertically before undergoing severe deformation at the edges as shown in Fig. 3. They also failed fairly often by losing adhesion at the edges, especially at the sharp corners. Initial wind tunnel tests also showed that a larger vertical deflection was desirable and it was

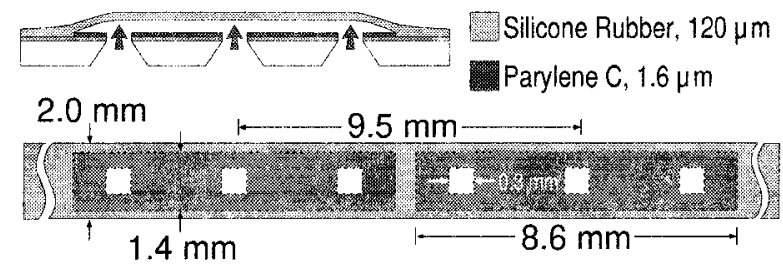

Fig. 2 First (2 mm) Balloon Actuator Design

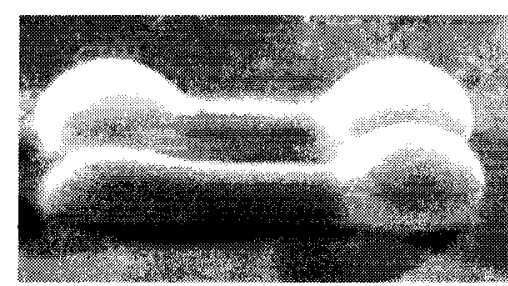

Fig. 3 Plastic Deformation of $1^{\text {st }}$ Generation Actuator

decided that a $3 \mathrm{~mm}$ wide actuator strip was desirable. The design was revised in three ways (Fig. 4) in terms of increasing reliability, both of the silicone itself, and the adhesion to the silicon frame. The width of exposed silicon on either side of the Parylene release layer was increased from $0.3 \mathrm{~mm}$ to $0.35 \mathrm{~mm}$. The corners of the release layer were rounded to decrease stress from the boundary conditions, and one inlet hole was removed while the remaining two were moved closer together to equalize the pressure exerted on the membrane.

This design has performed fairly well, but some small changes are still necessary. After extended cycling of

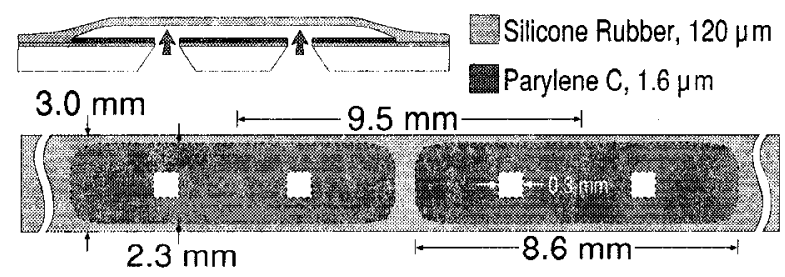

Fig. 4 Second (3 mm) Actuator Design to Reduce Plastic Deformation and Improve Adhesion the membranes, they fail along one edge with a pinhole implying that the silicone at the edges is undergoing high stress. A revised design would include changes in the boundary condition along the edge of the membrane to reduce the sharpness of the angle formed during actuation.

\section{FABRICATION}

The crucial fabrication hurdle is releasing a large area silicone membrane without damaging the material itself. Most typical sacrificial materials (oxide, silicon, photoresist, metal) are incompatible as the releasing etchants are harmful to silicone rubber after long term exposure.

While working with composite Parylene/silicone membranes, we discovered that silicone rubber has very poor adhesion to Parylene. Thus, a patterned layer of Parylene can be used as a release layer to form large area silicone membranes without the complications associated with etching a sacrificial layer.

The process is shown in Fig. 5. Starting with a $<100>$ silicon wafer, $0.5 \mu \mathrm{m}$ of LPCVD silicon nitride is deposited on both sides. The back side is patterned using RIE, and cavities are etched anisotropically using $\mathrm{KOH}$ forming $300 \mu \mathrm{m}$ square free-standing membranes. 1.6 $\mu \mathrm{m}$ of Parylene $\mathrm{C}$ is deposited on the front side and patterned using $\mathrm{O}_{2}$ plasma. Without removing the photoresist on the Parylene, the front side is roughened with $\mathrm{SF}_{6} / \mathrm{O}_{2}$ plasma. The photoresist is stripped and 100 to $150 \mu \mathrm{m}$ of MRTV-1 silicone rubber is spin-coated on the front side. The wafers are cured and the composite nitride/Parylene membranes are removed from the backside with $\mathrm{SF}_{6} / \mathrm{O}_{2}$ plasma followed by $\mathrm{O}_{2}$ plasma. Application of pressurized gas to the inlet holes frees the silicone membranes with very high yield.

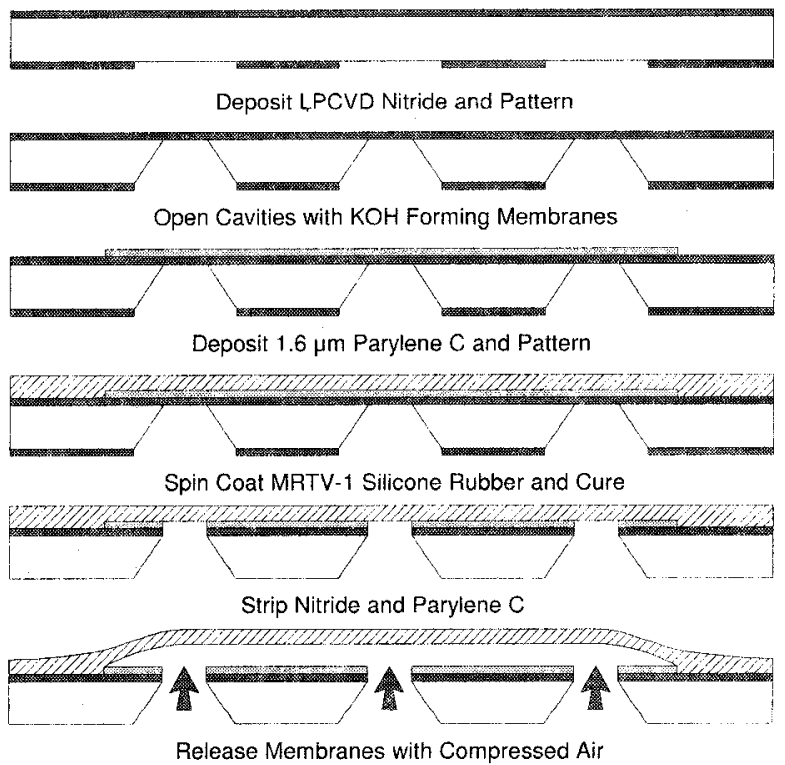

Fig. 5 Process Flow for Silicone Balloon Actuators 
The first devices made used MRTV-1 silicone rubber diluted with silicone fluid ( $8 \%$ by volume); a common practice to lower the viscosity. This mixture, while easier to spin coat with good results, degraded the mechanical properties of the cured silicone. Later devices used MRTV-1 diluted with a reactive silicone with superior results.

\begin{tabular}{|l|l|}
\hline Hardness, Durometer & Shore A 24 \\
\hline Tensile Strength, PSI & 500 \\
\hline Tear Strength, Die B 1b/in & 125 \\
\hline Tensile Elongation, \% & 1,000 \\
\hline Young's Modulus [4] & $0.5 \mathrm{MPa}$ \\
\hline Temperature Range, ${ }^{\circ} \mathrm{C}$ & -55 to 200 \\
\hline
\end{tabular}

Table 1 Mechanical Properties of MRTV-1 Silicone

\section{MECHANICAL TESTING}

Completed actuators were tested after fabrication to determine the pressure-deflection relationship using the experimental apparatus in Fig. 6. It was found that approximately 3 psi of pressure was necessary to free the silicone membranes from the substrate prior to testing, and any time that the membranes were allowed to "stick" to the substrate, a similar pressure was necessary, i.e. there was stiction between the silicone and Parylene. For the proposed usage as an aircraft control surface, this is not an issue and insures that the silicone surface stays relatively conformal when not actuated.

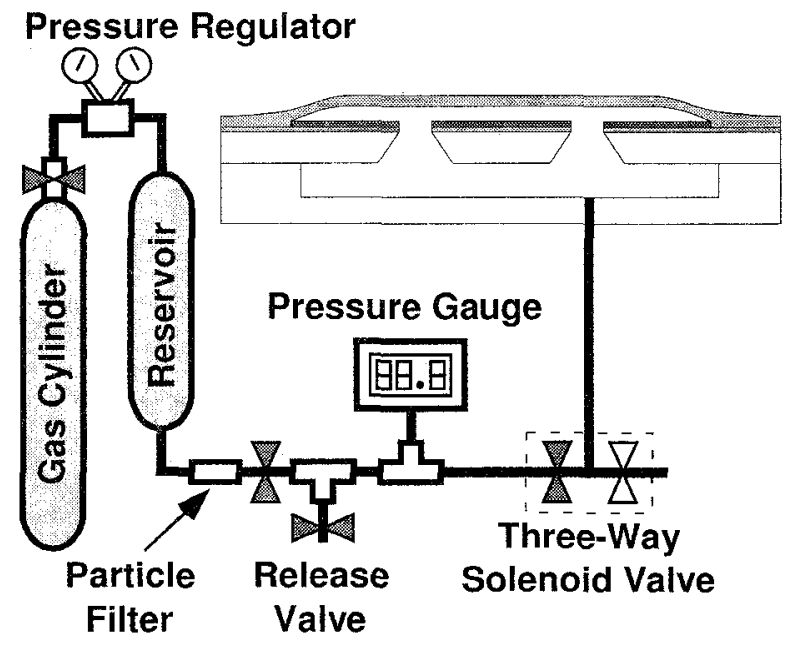

Fig. 6 Pressure vs. Deflection Apparatus

\section{Static Testing}

Due to the excessive plastic deformation of the first generation of actuators, extensive testing was limited to the second generation. Fig. 7 shows three sets of data for the same actuator taken at different times. The first (and lowest) trend line was taken directly after fabrication. At the low pressures used, no plastic

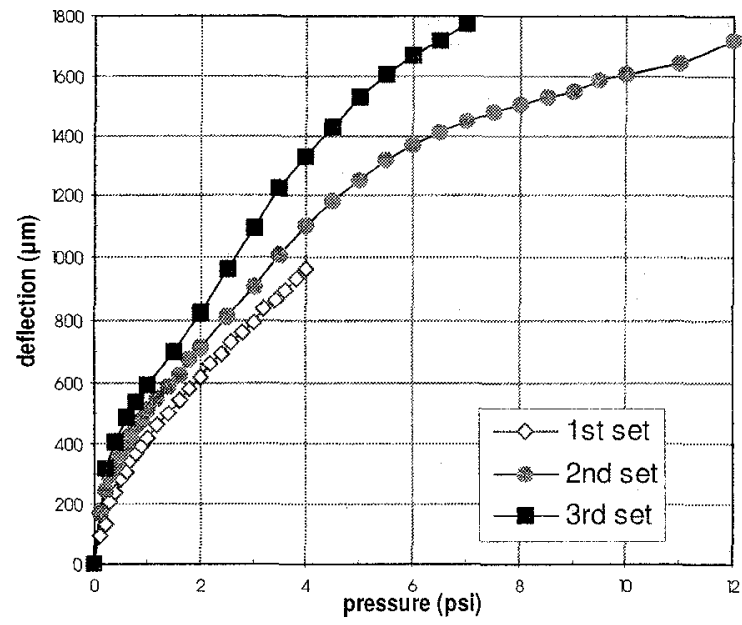

Fig. 7 Deflection vs. Pressure for the Same Actuator After Various Experiments

deformation was noted visually, or as a rapid slope change in the curve. The actuator was then inflated with a wide range of pressures up to $12 \mathrm{psi}$ to observe the behavior of the membrane (and generate the series in Fig. 8). A second set of data was taken and plastic deformation was observed at $10+$ psi although the "dog bone" shape exhibited by the first generation actuators wasn't noticed. The actuator was then tested past 15 psi and more plastic deformation was observed. A third set of data was then taken. At higher pressures, the deflection increased $350 \mu \mathrm{m}$ or more over the first set of data indicating permanent stretching of the silicone membrane and the "dog bone" shape was observed.

\section{Cyclic Testing}

To determine the reliability of the actuators under continuous use, eight actuators were cycled using a three-way solenoid valve at frequencies from one to two $\mathrm{Hz}$ at a pressure of 5.1 psi which was chosen to be out of the plastic regime. Pressure vs. deflection curves

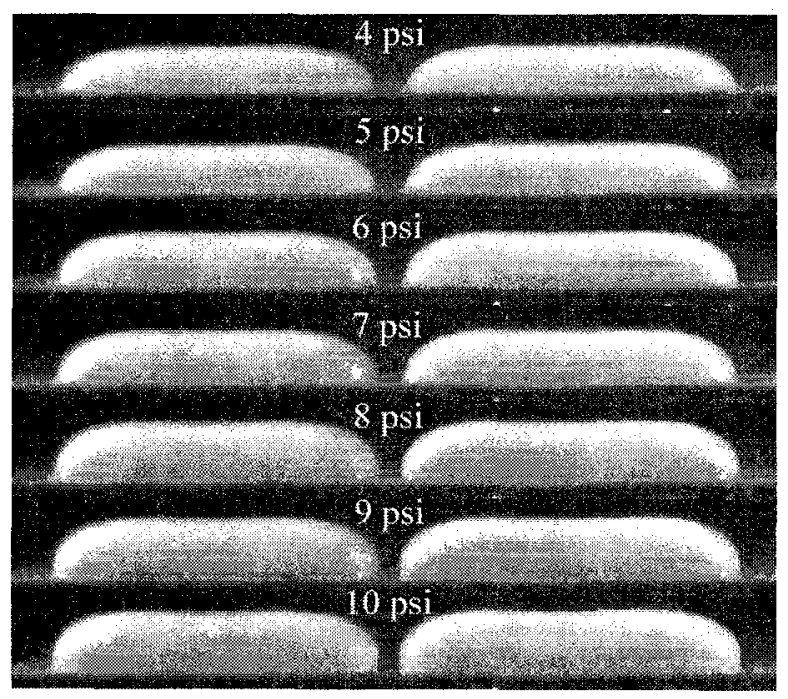

Fig. 8 Various States of Inflation for a $3 \mathrm{~mm}$ Actuator 


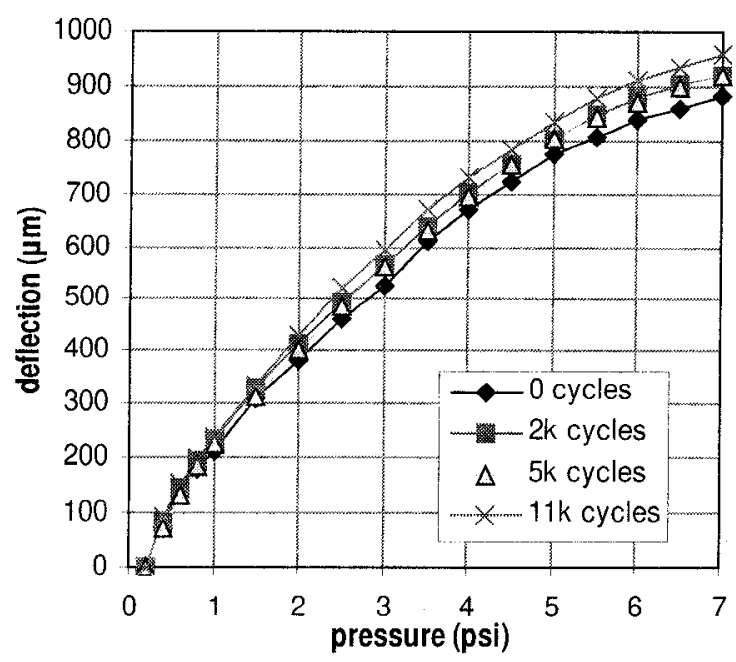

Fig. 9 Typical Deflection vs. Pressure Data for a Cycled Silicone Membrane Actuator

were taken after $0,2000,5000$, and 11000 cycles for each actuator. Deflection was recorded from an initial pressure of $0.2 \mathrm{psi}$ to take any slack out of the membrane and provide a consistent starting point.

Actuators failed at $4500,5000,7100,7900$, and 10000 cycles leaving three actuators after 11000 cycles. In each case failure was preceded by a slight peeling of the silicone at the edges of the Parylene release layer (at the point of highest stress during actuation) and failure consisted of a pinhole along the center of the long edge of the actuator. As the pressure was sufficiently low, none of the failures were due to bursting membranes.

Fig. 9 shows the pressure vs. deflection trend for one of three actuators that survived past 11,000 cycles (although the trend is similar for the actuators that failed). From 0 through 5,000 cycles, the curves are almost identical with a slight stretching occurring after 2,000 cycles. After 11,000 cycles, all three actuators showed signs of the silicone membrane losing adhesion at the edges and the higher deflection is partly due to this effect. The shape of the pressure vs. deflection curve doesn't change indicating that cyclic stress below the plastic region does not induce plasticity in the membrane which might lead to early membrane failure.

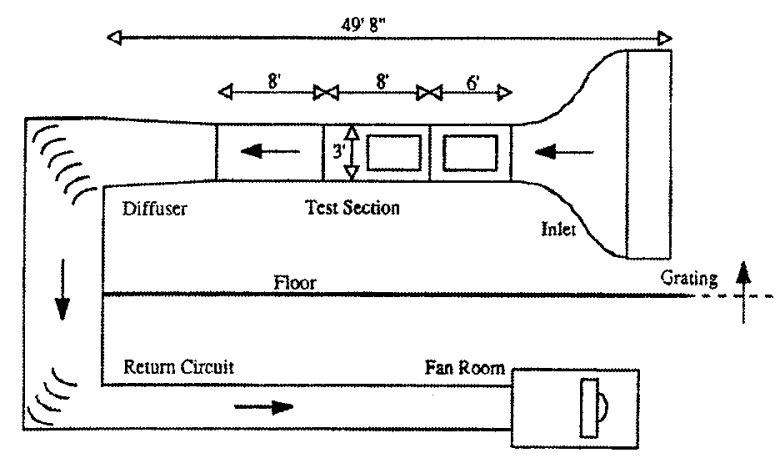

Fig. 10 UCLA Wind Tunnel

\section{WIND TUNNEL TESTING}

To verify the feasibility of using micro balloon actuators for control of a macro aerodynamic device such as a delta-wing aircraft, wind tunnel testing was performed. Theoretically, the similarity in scale of the actuators and the thin boundary layer before separation near the leading edge should allow the use of small perturbations to change the separation point. The development of leading edge vortices, which contribute a significant portion of the total lift at high angles of attack, are modified by this control. By manipulation of the vortex asymmetries, moments for wing motion control can be generated.

\section{Facility and Delta Wing Configuration}

An open-return wind tunnel was used for aerodynamic testing at UCLA (Fig. 10). The test-section is $91 \mathrm{~cm}$ by $91 \mathrm{~cm}$ by $730 \mathrm{~cm}$ long. The maximum operating speed of the wind tunnel is $45 \mathrm{~m} / \mathrm{s}$.

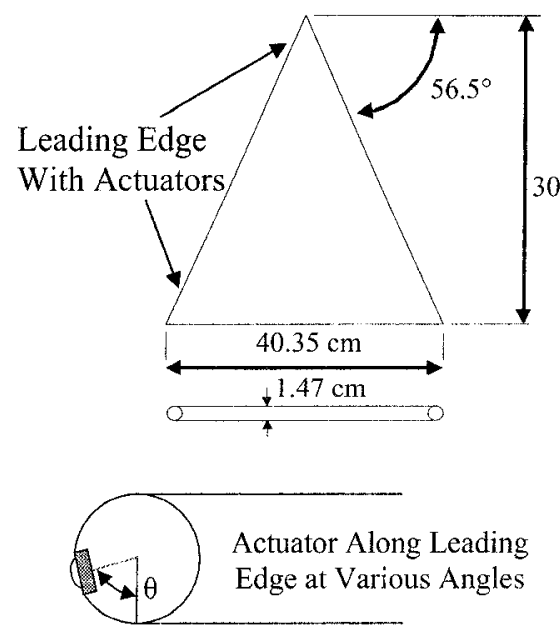

Fig. 11 Schematic of Delta Wing Model

A delta wing model (Fig. 11) with a swept angle of $56.5^{\circ}$ and chord length of $30 \mathrm{~cm}$ was employed. The delta wing model has round leading edges formed by a rod on which the micro balloon actuators are mounted. By rotating the rod, the position of the actuators can be varied over the entire leading edge area. A six component force/moment transducer (AMTI Inc.) was used to measure aerodynamic forces and moments. This transducer was used to record changes in the 3-axis torques caused by the micro balloons. Data was digitized and processed by a computer.

\section{Pitching, Yawing, and Rolling Moment Control}

To investigate the rolling moment generated by the two generations of actuators $(2 \mathrm{~mm}$ and $3 \mathrm{~mm}$ wide strips with respective vertical deflections of $1 \mathrm{~mm}$ and 1.6 $\mathrm{mm}$ ), actuators were positioned and actuated along one leading edge from the apex to the wing tip. Fig. 12 represents the effects of the $2 \mathrm{~mm}$ and $3 \mathrm{~mm}$ actuators 


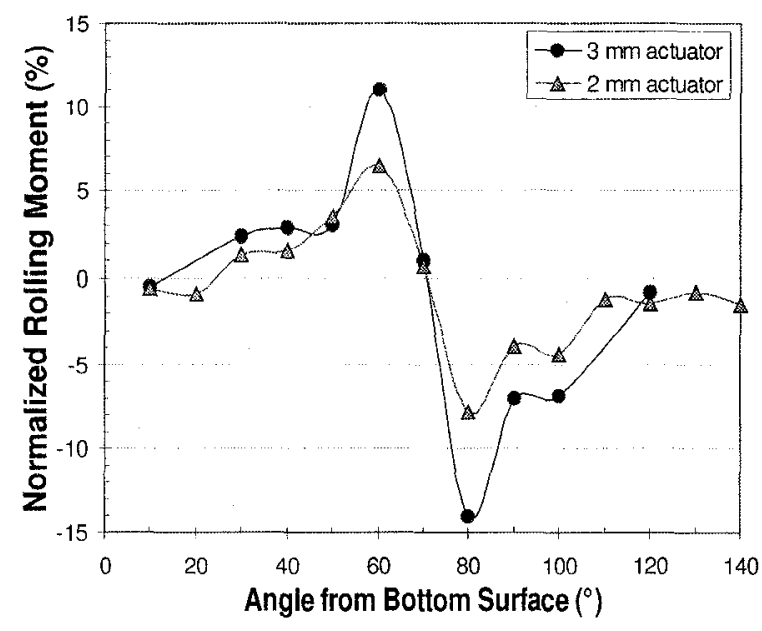

Fig. 12 Rolling Moment vs. Angular Location

on rolling moment at an angle of attack (AOA) of $25^{\circ}$. The data shows that $14 \%$ and $25 \%$ increments of the rolling moment can be obtained by two-sided actuation if we normalize these results to the experimentally obtained moment generated by a single leading-edge vortex.

In addition to rolling moments, we can also generate pitching and yawing with the same actuation. In order to compare the data with that generated by conventional control surfaces, like rudders, we plot the data for the 3 $\mathrm{mm}$ actuators as pitching, yawing, and rolling moment coefficients (Fig. 13). The pitching, yawing, and rolling moment coefficients, $\mathrm{C}_{\mathrm{p}}, \mathrm{C}_{\mathrm{y}}$, and $\mathrm{C}_{\mathrm{r}}$, are defined in the following way:

$$
\begin{aligned}
& C_{p}=\frac{\Delta M_{p}}{Q S c} \\
& C_{y}=\frac{\Delta M_{y}}{Q S b} \\
& C_{r}=\frac{\Delta M_{r}}{Q S b}
\end{aligned}
$$

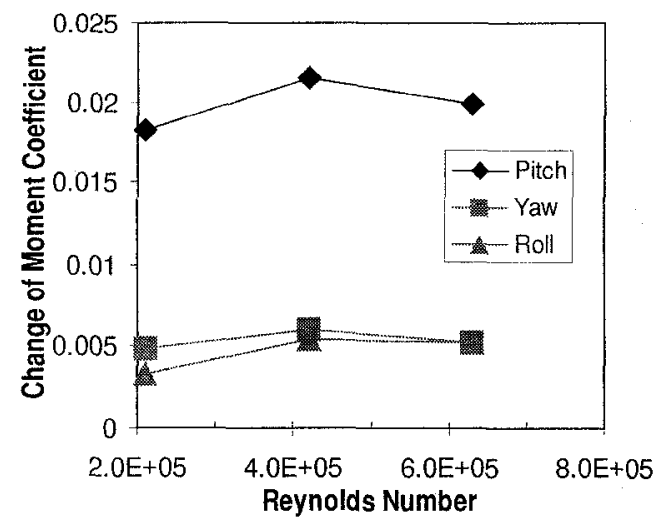

Fig. 13 Moment Coefficients vs. Reynolds Number at an Angle of Attack of $25^{\circ}$ where $\Delta M_{p}, \Delta M_{y}, \Delta M_{r}$ are the changes in the pitching, yawing, and rolling moments produced by micro actuation. $Q, S, b$, and $c$ are dynamic pressure, wing area, trailing edge length, and distance from the apex to the centroid of the wing respectively. From Fig. 13, we can see that pitching, yawing, and rolling moment coefficients are not sensitive to Reynolds number. The maximum pitching moment coefficient is about 0.02 . This value is large enough for most flight control.

\section{Flight Testing}

Several strips of $2 \mathrm{~mm}$ actuators (first generation) were attached to a small section of F-15 fighter jet wing and flight tested to assess their resistance to temperatures and pressures seen at high velocity. The section of wing (Fig. 14) also contained shear stress sensors which will be used in conjunction with the actuators for future flight control. The aircraft achieved a maximum speed of $0.9 \mathrm{Ma}$ and temperatures fluctuated from $-9{ }^{\circ} \mathrm{F}$ to $110^{\circ} \mathrm{F}$. It was found that all the MEMS devices survived the harsh flight test several times, which demonstrates the robustness of the devices.

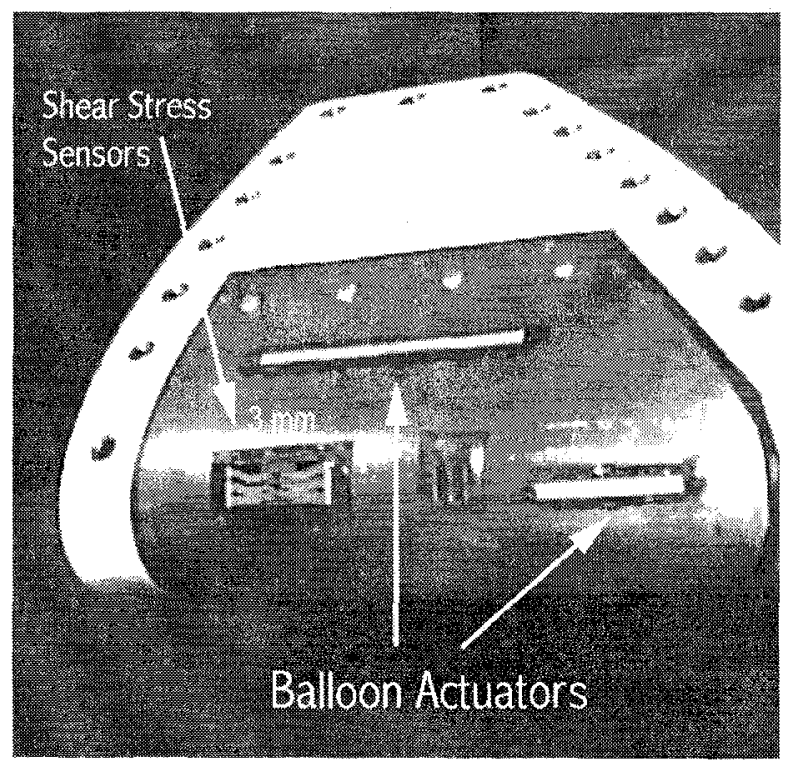

Fig. 14 Balloon Actuators and Shear Stress Sensors Attached to a Section of F-15 Wing

\section{CONCLUSION}

A novel, high yield fabrication process has been developed for silicone rubber balloon actuators. Arrays of large-deflection, large-force actuators for aerodynamic control have been fabricated. Balloon actuators have been tested under cyclic conditions to assess long-term reliability. Actuator strips mounted on the leading edge of a delta wing model have been characterized in a wind tunnel and the results indicate that they are suitable for use as aerodynamic control surfaces. Several strips of actuators have been flown successfully on an F-15 fighter jet demonstrating the 
durability of the actuators to elements encountered in transonic flight.

\section{ACKNOWLEDGEMENTS}

This work is supported under Navy Contract N6600197-C-8610. The authors would like to thank Xuan-Qi Wang for help with Parylene deposition and Trevor Roper for fabrication assistance.

\section{REFERENCES}

[1] C. Liu, T. Tsao, and Y. C. Tai, "Out of Plane Permalloy Magnetic Actuators for Delta-Wing Control," MEMS 1995, Amsterdam, The Netherlands, pp. 7-12 (1995).
[2] C. Vieider, O. Ohman and H. Elderstig, "A Pneumatically Actuated Microvalve with a Silicone Rubber Membrane for Integration with Fluid Handling Systems," Transducers 1995, Stockholm, Sweden, pp. 284-286 (1995).

[3] L. Bousse, E. Dijkstra, and O. Guenat, "HighDensity Arrays of Valves and Interconnects for Liquid Switching," Hilton Head 1996, Hilton Head Island, SC, pp. 272-275 (1996).

[4] X. Yang, C. Grosjean, Y. C. Tai, and C. M. Ho, "A MEMS Thermopneumatic Silicone Membrane Valve," MEMS 1997, Nagoya, Japan, pp. 114-118 (1997). 